\title{
Welche Rolle spielt Kompetenzdiagnostik im Rahmen von Lehr-Lernprozessen?
}

\author{
Susanne Bögeholz • Sabina Eggert
}

Zusammenfassung: Kompetenzdiagnostik kann Lehr-Lernprozesse unterstützen. Sie bezieht sich auf die Individual-, Klassen-, Schul- oder Länderebene und verfolgt damit jeweils spezifische Zwecke: So dienen Large-Scale-Assessments dem Bildungsmonitoring, während Assessments auf Individualebene entscheidend für persönliche Bildungswege sein können. In der Regel erfolgt Kompetenzdiagnostik mit Messmodellen der Item-Response-Theorie (IRT). Dies ist ein Grund, warum das Potenzial von Kompetenzdiagnostik für Feedback und individuelle Förderung bislang kaum ausgeschöpft wird. Lehrkräften fällt es vielfach schwer, Befunde aus IRT-Modellierungen zu interpretieren und für die Gestaltung von Lehr-Lernprozessen zu nutzen. Es ist daher ratsam, Lehrkräfte bei der Anwendung von Wissen aus Assessments für den Unterricht zu begleiten. Zudem sollte es Ziel sein, Unterricht und Kompetenzdiagnostik systematischer aufeinander zu beziehen. Kompetenzmodelle sollten die gemeinsame Referenz für das Unterrichten sowie für formatives und summatives Assessment sein. Prozessbegleitendes, formatives Assessment liefert eine Basis für kriteriales Feedback und kann dazu beitragen, die auf Systemebene definierten Standards in den Unterricht zu tragen. Dennoch bedarf es weiterer Forschung zur Kompetenzdiagnostik, deren Kommunikation und Einbeziehung für die Gestaltung von Lehr-Lernprozessen.

Schlüsselwörter: Kompetenzdiagnostik · Kompetenzmodell · Lehr-Lernprozesse · Formatives Assessment · Summatives Assessment

\section{Chances and challenges of competence assessment for teaching and learning}

\begin{abstract}
Competence assessment can support evidence-based teaching and learning. Depending on its specific purpose, competence assessment can be applied at the individual, the classroom or school level as well as on a national level. Large-scale-assessments are typical examples for system monitoring. Competence assessment on the individual level can provide important information with respect to individual qualification and learning outcomes. Typically, competence assessment uses psychometric models from Item-Response-Theory (IRT). However, until present, competence assessment still struggles to tap its full potential regarding feedback and individual support. Teachers often have difficulties to interpret results from competence assessments that are
\end{abstract}

(C) Springer Fachmedien Wiesbaden 2013

Prof. Dr. S. Bögeholz $(\bowtie) \cdot$ Dr. S. Eggert $(\bowtie)$

Biologische Fakultät, Georg-August-Universität Göttingen,

Waldweg 26, 37073 Göttingen, Deutschland

E-Mail: sboegeh@gwdg.de

Dr. S. Eggert

E-Mail: seggert1@gwdg.de 
based on IRT. Thus, it is crucial to support teachers in using these results to improve classroom teaching. One of the main challenges of educational measurement is to align everyday classroom teaching with formative and summative assessments and to establish competence models as a common ground. Process-orientated, formative assessment can inform criterion-based feedback and, consequently, promote teaching on the basis of educational standards that were defined on the system level. However, more work needs to be done with respect to research on assessments as well as their communication and implementation into teaching practice.

Keywords: Competence assessment · Competence model $\cdot$ Evidence-based teaching . Formative assessment $\cdot$ Summative assessment

Die Messung von Kompetenzen spielt auf nationaler Ebene spätestens seit dem wenig zufriedenstellenden Abschneiden deutscher Schüler/-innen in der internationalen Vergleichsstudie PISA 2000 eine zentrale Rolle (vgl. Deutsches PISA-Konsortium 2001). Seit Verabschiedung der nationalen Bildungsstandards - und der damit verbundenen Neuorientierung des Bildungssystems - wird Kompetenzdiagnostik sowohl im Rahmen der Evaluation und Normierung der nationalen Bildungsstandards in Deutschland (vgl. Walpuski et al. 2008; Kauertz et al. 2010) als auch auf Länderebene in Lernstandserhebungen und Vergleichsarbeiten systematisch durchgeführt (vgl. u. a. Leutner et al. 2007). Kompetenzdiagnostik trägt dazu bei, intensiver auf die mittel- und langfristig wirkenden Lehr-Lernprozesse zu fokussieren. Das heißt es geht darum, den Erwerb von Kompetenzen gemäß der Standards langfristig über Unterrichtsentwicklung zu unterstützen (vgl. z. B. EMSE-Netzwerk 2008; KMK 2010).

Die Ziele von Kompetenzdiagnostik können dabei sehr unterschiedlich sein. Im ursprünglichen Sinne bezieht sich Kompetenzdiagnostik auf die Individualebene und dient der „Vorbereitung von Entscheidungen im Einzelfall“ (Leutner et al. 2007, S. 151). Im Gegensatz dazu ermöglicht Kompetenzdiagnostik im Rahmen von Vergleichsarbeiten und Lernstandserhebungen in der Regel Aussagen auf aggregierter Klassen-, Schul- oder Länderebene (vgl. u. a. Leutner et al. 2007).

Die unterschiedlichen Arten der Kompetenzdiagnostik verfolgen unterschiedliche Zwecke und erlauben schließlich auch nur für diese Zwecke spezifische Aussagen und Rückmeldungen (vgl. Koeppen et al. 2008). Während Kompetenzdiagnostik im Rahmen von summativen Large-Scale-Assessments ein Bildungsmonitoring ermöglicht (vgl. Leutner et al. 2008), erlaubt summatives Assessment auf Individualebene Aussagen über die Kompetenz der Schüler/innen zu einem bestimmten Zeitpunkt (vgl. Leutner et al. 2007; Koeppen et al. 2008). Die Performanz in derartigen Tests auf Individualebene kann weitreichende Konsequenzen für den weiteren Bildungsweg haben (vgl. ebd.). Kompetenzen im Rahmen summativer Assessments werden in der Regel mit Hilfe von Messmodellen der Item-Response-Theorie (IRT) modelliert (vgl. Hartig und Frey 2013 in diesem Heft).

Inwiefern die IRT-Methodik im Rahmen von Kompetenzdiagnostik unmittelbar oder mittelbar für den Unterricht und dabei vor allem als Feedback-Instrument für Lehrkräfte verwendet werden kann, ist eine zentrale Herausforderung für die Forschung. So werden Daten aus Vergleichsarbeiten auf Klassenebene an Lehrkräfte zurückgemeldet, um ihnen ein kriteriales Feedback zur Reflexion und Optimierung ihres eigenen Unterrichts zu geben (Leutner et al. 2007). Das alleinige Bereitstellen derartiger Informationen hat 
jedoch kaum Einfluss auf die Verbesserung der Unterrichtsqualität. Zentral ist es, dass die Lehrkräfte die Verfahrensweise ernst nehmen und als praktikabel sowie nützlich einstufen (vgl. Bonsen et al. 2006; zur Begegnung negativer Auswirkungen von Kompetenzdiagnostik siehe McElvany und Rjosk 2013 in diesem Heft). Erfolgversprechender scheinen daher (fach-)didaktische Ansätze zu sein, die auf Basis von Kompetenzdiagnostik den Lehrkräften helfen, ihren Unterricht zu verbessern und die in ein umfangreiches Fortbildungskonzept eingebunden sind (vgl. Maier 2008). Anglo-amerikanische Studien zeigen auch, dass die Ergebnisse von Kompetenzmessungen von Lehrkräften noch nicht als unmittelbar hilfreich für den Unterricht wahrgenommen werden (vgl. Koeppen et al. 2008). Dies hat mehrere Gründe, darunter die zur klassischen Notengebung unterschiedliche Herangehensweise bei der Skalierung von Kompetenzen im Rahmen der IRT-Modellierung und die damit verbundene teilweise ungewohnte Darstellung und schwierige Vermittelbarkeit der Ergebnisse (vgl. Hartig und Frey 2013 in diesem Heft).

Derzeitige Studien zur Kompetenzdiagnostik schöpfen ihr Potenzial als Unterstützungs- und Feedbackinstrumente für Lehrkräfte bislang noch kaum aus. Ziel muss es sein, Kompetenzdiagnostik und Unterricht stärker aufeinander zu beziehen. Zur Umsetzung wäre es erforderlich, die diagnostische Kompetenz von Lehrkräften noch stärker und systematischer in Fortbildungsprogrammen zu entwickeln. Dazu zählt beispielsweise, Lernvoraussetzungen und Lernprozesse bei Schülerinnen und Schülern diagnostizieren zu können (vgl. Kompetenz 7 in KMK 2004).

Darüber hinaus können auch Kompetenzstrukturmodelle, wie es sie beispielsweise für die naturwissenschaftlichen Fächer, die Mathematik oder aber die Sprachen bereits gibt, noch stärker für den Unterricht nutzbar gemacht werden (vgl. Pant 2013 in diesem Heft). Dies kann geschehen, indem Lehrkräfte ihren Unterricht an den vorliegenden Kompetenzmodellen ausrichten (vgl. Eggert et al. 2010; vgl. auch Tesch et al. 2008). Dabei können Unterrichts- und Schulentwicklungsprojekte, die Forschung und Praxis verbinden, ein Instrument für eine erfolgreiche Integration von Kompetenzmodellen in den Unterricht darstellen. Beispiele hierfür sind „Biologie im Kontext“ (Bayrhuber et al. 2007) sowie der Hamburger Schulversuch ,alles $>>$ könner“ in Kombination mit dem Forschungsprogramm „komdif“ (Harms und Möller 2012). Des Weiteren können Diagnoseaufgaben von Lehrkräften eingesetzt und nach Schulung auch von ihnen ausgewertet werden (vgl. „Science Education for Public Understanding Project“; Wilson und Sloane 2000). Eine IRT-Modellierung kann auf Basis der Lehrerauswertungen durch Forschungsinstitute vorgenommen werden. Denkbar ist hier auch eine Verbindung von Auswertung und IRTModellierung durch technologiebasierte Assessment- und Feedbacksysteme (vgl. Jurecka 2008; Koeppen et al. 2008; zu Vor- und Nachteilen von computerbasierten Verfahren siehe Frey und Hartig 2013 in diesem Heft).

Ein weiteres zentrales Element zur Vernetzung von Kompetenzdiagnostik und Unterricht ist der verstärkte Einsatz formativen Assessments als Ergänzung zum summativen Assessment. Formatives Assessment auf Individualebene gibt Aufschluss über Lernprozesse sowie Stärken und Schwächen und kann darauf aufbauend Potenziale für individuelle Förderungsmöglichkeiten aufzeigen (vgl. u. a. Koeppen et al. 2008; Harks et al. im Druck). Neuere Studien konnten zeigen, dass prozess-orientiertes Feedback als nützlicher und als kompetenzunterstützender wahrgenommen wird als sozial-vergleichendes Feedback (vgl. Rakoczy et al. 2011). Darüber hinaus zeigt eine Laborstudie unter Nut- 
zung von Kompetenzstufenmodellen, dass ,kompetenzbezogene Rückmeldung, vermittelt über ihre wahrgenommene Nützlichkeit einen positiveren Effekt auf Motivation und Leistungsänderung [hat] als Rückmeldung in Form von Noten“ (Harks et al. im Druck, S. 21). Formatives Assessment zeichnet sich durch in den Prozess einfließende Rückmeldungen aus. Es kann dazu beitragen, auf Systemebene definierte Standards in den Unterricht zu transportieren (vgl. Maier 2008). Existierende Feedbackmodelle können dabei als Grundlage zur Konzeption von Feedback sowohl für Lehrkräfte als auch für Schüler/-innen genutzt werden (vgl. Hattie und Timperley 2007; Rakoczy et al. 2008).

Abschließend lässt sich erstens festhalten, dass Kompetenzdiagnostik aus mehreren Komponenten bestehen sollte, die sich ergänzen und Synergien aufweisen: So sind Diagnose von Schülerkompetenzen in täglichen Lehr-Lernarrangements und Diagnostik im Rahmen von summativen Assessments komplementär zueinander. Wichtig bei Kompetenzdiagnostik - insbesondere mit Blick auf die Optimierung von Lehr-Lernprozessen - ist, dass die verwendeten Diagnoseinstrumente von den Beteiligten verstanden werden (vgl. Wilson 2008 sowie Bonsen et al. 2006). Idealerweise basieren zweitens formative und summative Assessments in einem bestimmten Kompetenzbereich auf denselben theoretischen Annahmen, d. h. auf den gleichen Kompetenzmodellen. Diese Kompetenzmodelle sagen idealiter auch etwas über die Entwicklung von Kompetenzen aus (vgl. Robitzsch 2013 in diesem Heft). Festzuhalten ist drittens, dass Kompetenzdiagnostik eine wichtige Rolle für Lehr-Lernprozesse spielen kann. Desiderata sind derzeit - neben Forschungen zur Entwicklung von Schülerkompetenzen und zur Entwicklung diagnostischer Kompetenzen von Lehrkräften - Forschungen, um herauszufinden, wie das Potenzial von formativen und summativen Assessments für dessen Nutzung durch Lehrkräfte zur Weiterentwicklung von Unterricht möglichst optimal ausgeschöpft werden kann.

Danksagung: Diese Veröffentlichung wurde ermöglicht durch Sachbeihilfen der Deutschen Forschungsgemeinschaft (Kennz.: BO 1730/3-2) im Schwerpunktprogramm „Kompetenzmodelle zur Erfassung individueller Lernergebnisse und zur Bilanzierung von Bildungsprozessen“" (SPP 1293).

\section{Literatur}

Bayrhuber, H., Bögeholz, S., Elster, D., Hammann, M., Hößle, C., Lücken, M., Mayer, J., Nerdel, C., Neuhaus, B., Prechtl, H., \& Sandmann, A. (2007). Biologie im Kontext - Ein Programm zur Kompetenzförderung durch Kontextorientierung im Biologieunterricht und zur Unterstützung von Lehrerprofessionalisierung. Der mathematische und naturwissenschaftliche Unterricht, 60, 282-286.

Bonsen, M., Büchter, A., \& Peek, R. (2006). Datengestützte Schul- und Unterrichtsentwicklung: Bewertungen der Lernstandserhebungen in NRW durch Lehrerinnen und Lehrer. Jahrbuch der Schulentwicklung, 14, 125-148.

Deutsches PISA-Konsortium. (2001). PISA 2000: Basiskompetenzen von Schülerinnen und Schülern im internationalen Vergleich. Opladen: Leske + Budrich.

Eggert, S., Bögeholz, S., Watermann, R., \& Hasselhorn, M. (2010). Förderung von Bewertungskompetenz im Biologieunterricht durch zusätzliche metakognitive Strukturierungshilfen beim Kooperativen Lernen - Ein Beispiel für Veränderungsmessung. Zeitschrift für die Didaktik der Naturwissenschaften, 16, 299-314. 
EMSE-Netzwerk [empiriegestützte Schulentwicklung EMSE]. (2008). Nutzung und Nutzen von Schulrückmeldungen im Rahmen standardisierter Lernstandserhebungen/Vergleichsarbeiten. Zweites Positionspapier des EMSE-Netzwerkes - verabschiedet auf der 9. EMSE-Fachtagung am 16.-17. Dezember 2008 in Nürnberg. http://www.emse†netzwerk.de/uploads/Main/ EMSE_Positionsp2_Rueckmeldungen.pdf. Zugegriffen: 28.Okt. 2012.

Frey, A., \& Hartig, J. (2013). Wann sollten computerbasierte Verfahren zur Messung von Kompetenzen anstelle von papier- und bleistift-basierten Verfahren eingesetzt werden? In D. Leutner, E. Klieme, J. Fleischer \& H. Kuper (Hrsg.), Kompetenzmodelle zur Erfassung individueller Lernergebnisse und zur Bilanzierung von Bildungsprozessen: aktuelle Diskurse im DFGSchwerpunktprogramm (18. Sonderheft der Zeitschrift für Erziehungswissenschaft, DOI: 10.1007/s11618-013-0385-1.) Wiesbaden: VS Verlag für Sozialwissenschaften.

Harks, B., Rakoczy, K., Klieme, E., Hattie, J., \& Besser, M. (im Druck). Indirekte und moderierte Effekte von Rückmeldung auf Leistung und Motivation. In H. Ditton \& A. Müller (Hrsg.), Rückmeldungen: Theoretische Grundlagen, empirische Befunde, praktische Anwendungsfelder. München: Waxmann.

Harms, U., \& Möller, J. (2012). Forschung im Rahmen eines Schulversuchs: Exemplarische Arbeiten aus dem Forschungsprogramm komdif. Unterrichtswissenschaft, 40, 195-196.

Hartig, J., \& Frey, A. (2013). Sind Modelle der Item-Response-Theorie (IRT) das „Mittel der Wahl“ für die Modellierung von Kompetenzen? In D. Leutner, E. Klieme, J. Fleischer \& H. Kuper (Hrsg.), Kompetenzmodelle zur Erfassung individueller Lernergebnisse und zur Bilanzierung von Bildungsprozessen: aktuelle Diskurse im DFG-Schwerpunktprogramm (18. Sonderheft der Zeitschrift für Erziehungswissenschaft, DOI: 10.1007/s11618-013-0386-0.) Wiesbaden: VS Verlag für Sozialwissenschaften.

Hattie, J., \& Timperley, H. (2007). The power of feedback. Review of Educational Research, 77, $81-112$.

Jurecka, A. (2008). Introduction to the computer-based assessment of competencies. In J. Hartig, E. Klieme \& D. Leutner (Hrsg.), Assessment of competencies in educational contexts (S. 193213). Göttingen: Hogrefe.

Kauertz, A., Fischer, H. E., Mayer, J., Sumfleth, E., \& Walpuski, M. (2010). Standardbezogene Kompetenzmodellierung in den Naturwissenschaften der Sekundarstufe I. Zeitschrift für Didaktik der Naturwissenschaften, 16, 135-153.

KMK (2004) = Sekretariat der Ständigen Konferenz der Kultusminister der Länder in der Bundesrepublik Deutschland. (2004). Standards für die Lehrerbildung: Bildungswissenschaften. Beschluss der Kultusministerkonferenz vom 16.12.2004. http://www.kmk.org/fileadmin/veroeffentlichungen_beschluesse/2004/2004_12_16-Standards-Lehrerbildung.pdf. Zugegriffen: 28.Okt. 2012.

KMK (2010) = Sekretariat der Ständigen Konferenz der Kultusminister der Länder in der Bundesrepublik Deutschland. (2010). Konzeption der Kultusministerkonferenz zur Nutzung der Bildungsstandards für die Unterrichtsentwicklung. Köln: Wolters-Kluwer.

Koeppen, K., Hartig, J., Klieme, E., \& Leutner, D. (2008). Current issues in competence modelling and assessment. Zeitschrift für Psychologie/Journal of Psychology, 216, 61-73.

Leutner, D., Fleischer, J., Spoden, C., \& Wirth, J. (2007). Landesweite Lernstandserhebungen zwischen Bildungsmonitoring und Individualdiagnostik. In M. Prenzel, I. Gogdin \& H. - H. Krüger (Hrsg.), Kompetenzdiagnostik (8. Sonderheft der Zeitschrift für Erziehungswissenschaft, S. 149-167.) Wiesbaden: VS Verlag für Sozialwissenschaften.

Leutner, D., Hartig, J., \& Jude, N. (2008). Measuring competencies: Introduction to concepts and questions of assessment in education. In J. Hartig, E. Klieme \& D. Leutner (Hrsg.), Assessment of competencies in educational contexts (S. 177-192). Göttingen: Hogrefe.

Maier, U. (2008). Vergleichsarbeiten im Vergleich - Akzeptanz und wahrgenommener Nutzen standardbasierter Leistungsmessungen in Baden-Württemberg und Thüringen. Zeitschrift für Erziehungswissenschaft, 8(3), 453-474. 
McElvany, N., \& Rjosk, C. (2013). Wann kann Kompetenzdiagnostik negative Auswirkungen haben? In D. Leutner, E. Klieme, J. Fleischer \& H. Kuper (Hrsg.), Kompetenzmodelle zur Erfassung individueller Lernergebnisse und zur Bilanzierung von Bildungsprozessen: aktuelle Diskurse im DFG-Schwerpunktprogramm (18. Sonderheft der Zeitschrift für Erziehungswissenschaft, DOI: 10.1007/s11618-013-0388-y). Wiesbaden: VS Verlag für Sozialwissenschaften.

Pant, H. A. (2013). Wer hat einen Nutzen von Kompetenzmodellen? In D. Leutner, E. Klieme, J. Fleischer \& H. Kuper (Hrsg.), Kompetenzmodelle zur Erfassung individueller Lernergebnisse und zur Bilanzierung von Bildungsprozessen: aktuelle Diskurse im DFG-Schwerpunktprogramm (18. Sonderheft der Zeitschrift für Erziehungswissenschaft, DOI: 10.1007/ s11618-013-0387-z). Wiesbaden: VS Verlag für Sozialwissenschaften.

Rakoczy, K., Klieme, E., Bürgermeister, A., \& Harks, B. (2008). The interplay between student evaluation and instruction. Zeitschrift für Psychologie/Journal of Psychology, 216, 111-124.

Rakoczy, K., Harks, B., Bürgermeister, A., \& Klieme, E. (2011, August-September). The impact of process-oriented feedback on learning in mathematics - mediated by students' individual perception and moderated by goal orientation? Paper presented at the 14th Biennial Conference of the European Association of Research on Learning and Instruction (Earli), Exeter, UK.

Robitzsch, A. (2013). Wie robust sind Struktur- und Niveaumodelle? Wie zeitlich stabil und über Situationen hinweg konstant sind Kompetenzen? Zeitschrift für Erziehungswissenschaft. In D. Leutner, E. Klieme, J. Fleischer \& H. Kuper (Hrsg.), Kompetenzmodelle zur Erfassung individueller Lernergebnisse und zur Bilanzierung von Bildungsprozessen: aktuelle Diskurse im DFG-Schwerpunktprogramm (18. Sonderheft der Zeitschrift für Erziehungswissenschaft, DOI: 10.1007/s11618-013-0383-3). Wiesbaden: VS Verlag für Sozialwissenschaften.

Tesch, B., Leupold, E., \& Köller, O. (2008). Bildungsstandards Französisch: konkret/ Sekundarstufe I: Grundlagen, Aufgabenbeispiele und Unterrichtsanregungen. Berlin: IQB/Cornelsen Scriptor.

Walpuski, M., Kampa, N., Kauertz, A., \& Wellnitz, N. (2008). Evaluation der Bildungsstandards in den Naturwissenschaften. Der mathematische und naturwissenschaftliche Unterricht, 61, 323-326.

Wilson, M. (2008). Cognitive diagnosis using item response models. Zeitschrift für Psychologie/ Journal of Psychology, 216, 74-88.

Wilson, M., \& Sloane, K. (2000). From principles to practice. An embedded assessment system. Applied Measurement in Education, 13, 181-208. 